\title{
Dietary intake in the Personalized Medicine Research Project: a resource for studies of gene-diet interaction
}

\author{
Lacie Strobush ${ }^{1}$, Richard Berg², Deanna Cross ${ }^{1}$, Wendy Foth', Terrie Kitchner ${ }^{1}$, Laura Coleman³, \\ Catherine A McCarty ${ }^{1 *}$
}

\begin{abstract}
Background: To describe the dietary intake of participants in the Personalized Medicine Research Project (PMRP), and to quantify differences in nutrient intake by smoking status and APOE4-a genetic marker that has been shown to modify the association between risk factors and outcomes.
\end{abstract}

Methods: The PMRP is a population-based DNA, plasma and serum biobank of more than 20,000 adults aged 18 years and older in central Wisconsin. A questionnaire at enrollment captures demographic information as well as self-reported smoking and alcohol intake. The protocol was amended to include the collection of dietary intake and physical activity via self-reported questionnaires: the National Cancer Institute 124-item Diet History Questionnaire and the Baecke Physical Activity Questionnaire. These questionnaires were mailed out to previously enrolled participants. APOE was genotyped in all subjects.

Results: The response rate to the mailed questionnaires was $68.2 \%$ for subjects who could still be contacted (alive with known address). Participants ranged in age from 18 to 98 years (mean 54.7) and 61\% were female. Dietary intake is variable when comparing gender, age, smoking, and APOE4. Over 50\% of females are dietary supplement users; females have higher supplement intake than males, but both have increasing supplement use as age increases. Food energy, total fat, cholesterol, protein, and alcohol intake decreases as both males and females age. Female smokers had higher macronutrient intake, whereas male nonsmokers had higher macronutrient intake. Nonsmokers in both genders use more supplements. In females, nonsmokers and smokers with APOE4 had higher supplement use. In males, nonsmokers with APOE4 had higher supplement use between ages 18-39 only, and lower supplement use at ages above 39. Male smokers with APOE4 had lower supplement use.

Conclusion: Dietary intake in PMRP subjects is relatively consistent with data from the National Health and Nutrition Examination Survey (NHANES). Findings suggest a possible correlation between the use of supplements and APOE4. The PMRP dietary data can benefit studies of gene-environment interactions and the development of common diseases.

\section{Background}

With the completion of the Human Genome Project, the laboratory tools to quantify genetic variation in human populations exist. Analyzing genetic variation could lead to the discovery of genetic predictors of disease. In addition to those predictors, it is important to

\footnotetext{
* Correspondence: mccarty.catherine@mcrf.mfldclin.edu

'Center for Human Genetics, Marshfield Clinic Research Foundation, 1000 N. Oak Ave (MLR), Marshfield, WI 54449, USA

Full list of author information is available at the end of the article
}

quantify gene-environment interactions that modify genetic associations. Dietary intake is associated with multiple health outcomes and is one of the critical, potentially modifiable, environmental exposures to consider in gene-environment studies [1]. Food frequency questionnaires (FFQ) are the most cost-effective tool to measure usual dietary intake in large cohort studies, but caution must be taken with the interpretation and use of macronutrient data from FFQ [1]. Interactions involving alcohol intake as an environmental factor have
C Biomed Central 
been studied to illustrate its impact on development of certain health outcomes [2]. Another common, modifiable, environmental risk factor for consideration in gene-environment studies is smoking; dietary intake has been shown to vary by smoking status [3].

Apolipoprotein E (APOE) is one of the most commonly researched genes in studies of gene-environment interactions. Through its function as a ligand and its involvement with chylomicrons, very-low density lipoproteins (VLDL), and high-density lipoproteins (HDLs), APOE helps maintain cholesterol and fat levels in the body [4]. The APOE gene has three alleles, one of which is E4. The E4 allele has been associated with both coronary heart disease (CHD) and early onset of Alzheimer's disease [5]. Total cholesterol and LDL cholesterol levels in general are highest in people who have an E4 allele [6,7]. Some studies have suggested that APOE4 carriers who are smokers are at increased risk for coronary heart disease compared to non-smokers [5].

The Personalized Medicine Research Project (PMRP) is a population-based DNA, plasma and serum biobank designed to facilitate genetic epidemiology and pharmacogenetic studies [8-11]. The comprehensive medical record of the Marshfield Clinic is ideal for the identification of affected cases and appropriate controls; however, limited information about personal exposure is collected in a standardized fashion in the context of routine clinical care. Therefore, assessments of known, potentially modifiable, risk factors for disease were included in the study protocol. They include smoking status, alcohol intake, and a detailed FFQ and physical activity questionnaire. The purpose of this paper is to describe the PMRP biobank as a resource for gene-diet studies, to quantify the extent to which smoking status, alcohol consumption, and the APOE genotype are associated with dietary intake in the population, and to explain how these factors may need to be considered as covariants in future gene-nutrient studies.

\section{Methods}

\section{Personalized Medicine Research Project (PMRP)}

Details of the PMRP have been published previously [8-11]. In summary, the project was designed to establish a large biobank consisting of DNA, plasma and serum from a large representative sample. Since central Wisconsin has a relatively stable population and the majority of residents receive care at a Marshfield Clinic, the geographic area is ideal for research over a long period of time. Participants that were invited were residents of at least 18 years of age, living in one of 19 zip-codes surrounding Marshfield, WI, and the vast majority received most of their medical care in the Marshfield Clinic system. After subjects have signed the written consent form, which allows access to their comprehensive Marshfield
Clinic medical record, subjects complete a brief questionnaire about demographics, smoking status, alcohol intake, and health history. DNA, plasma, and serum samples were extracted and stored from whole blood. To extract the DNA, the Gentra's AUTOPURE ${ }^{\circledR}$ system was used. White blood cells were isolated and lysed; through multiple steps of centrifugation and decanting, DNA was obtained, washed and stored at $-80^{\circ} \mathrm{C}$ [8]. All procedures were reviewed and approved by the Marshfield Clinic Institutional Review Board.

\section{Quantification of dietary intake}

The study protocol was amended after nearly 18,000 subjects were enrolled in PMRP to include usual dietary intake and physical activity. Usual dietary intake was measured using the validated National Cancer Institute 124-item Diet History Questionnaire (DHQ) [12-17]. For those subjects already enrolled, the DHQ was mailed out, with a second mailing and follow-up phone calls as needed to increase the participation rate. The completed questionnaires were scanned and nutrient files were created using the software package Diet"Calc (http://riskfactor.cancer.gov/DHQ/dietcalc/). Questionnaires with more than half of the pages or items not complete were excluded from analysis. Standard units were used. ATE CSFII refers to the units for vitamin E. CSFII stands for Continuing Survey of Food Intakes by Individuals; ATE stands for alpha-tocopherol equivalent, which is a form of vitamin $\mathrm{E}$ absorbed by humans. IU and RE refer to the units for measuring vitamin A. IU stands for international units, and RE stands for retinol equivalents.

\section{Quantification of APOE4 genotype}

Matrix-assisted laser desorption/ionization time-of-flight (MALDI-TOF) mass spectrometry was used to genotype the polymorphisms of the APOE gene. PCR reactions, which used primers designed by the Assay Designer 2.05software from Sequenom, amplified designated regions of DNA. Primer extension reactions were performed to generate allele-specific products that are one base longer than the original primer. The products were placed onto a matrix arrayed silicon chip and analyzed by a MALDITOF mass spectrometer and Sequenom SpectroTYPER 3.4 software. The mass spectrometer determines alleles based on different molecular weights [11].

\section{Statistical analyses}

Preliminary analyses included tabular and graphical summaries describing participant demographics, dietary intake, and APOE genotypes. In the primary study analyses we analyze dietary intake for associations with body size (BMI), smoking status and APOE4, while controlling for expected age and gender differences. 
Stratification and graphical displays were used to investigate the plausibility and consistency of potential associations, and to identify potential interactions and confounding. Group comparisons of dietary intake stratified by gender and age were conducted using rankbased methods (Wilcoxon and Kruskal-Wallis tests). Analyses were conducted using SAS ${ }^{\mathbb{B}}$ (version 9.2, SAS Inc., Cary, NC). Results were considered statistically significant at the $5 \%$ level $(\mathrm{p}<0.05)$ without adjustment for multiple comparisons.

\section{Results}

The response rate to the mailed questionnaires was $62.8 \%$ for subjects who could still be contacted (alive with known address). Approximately $3 \%$ of participants could not be located and $4 \%$ were deceased. Figure 1

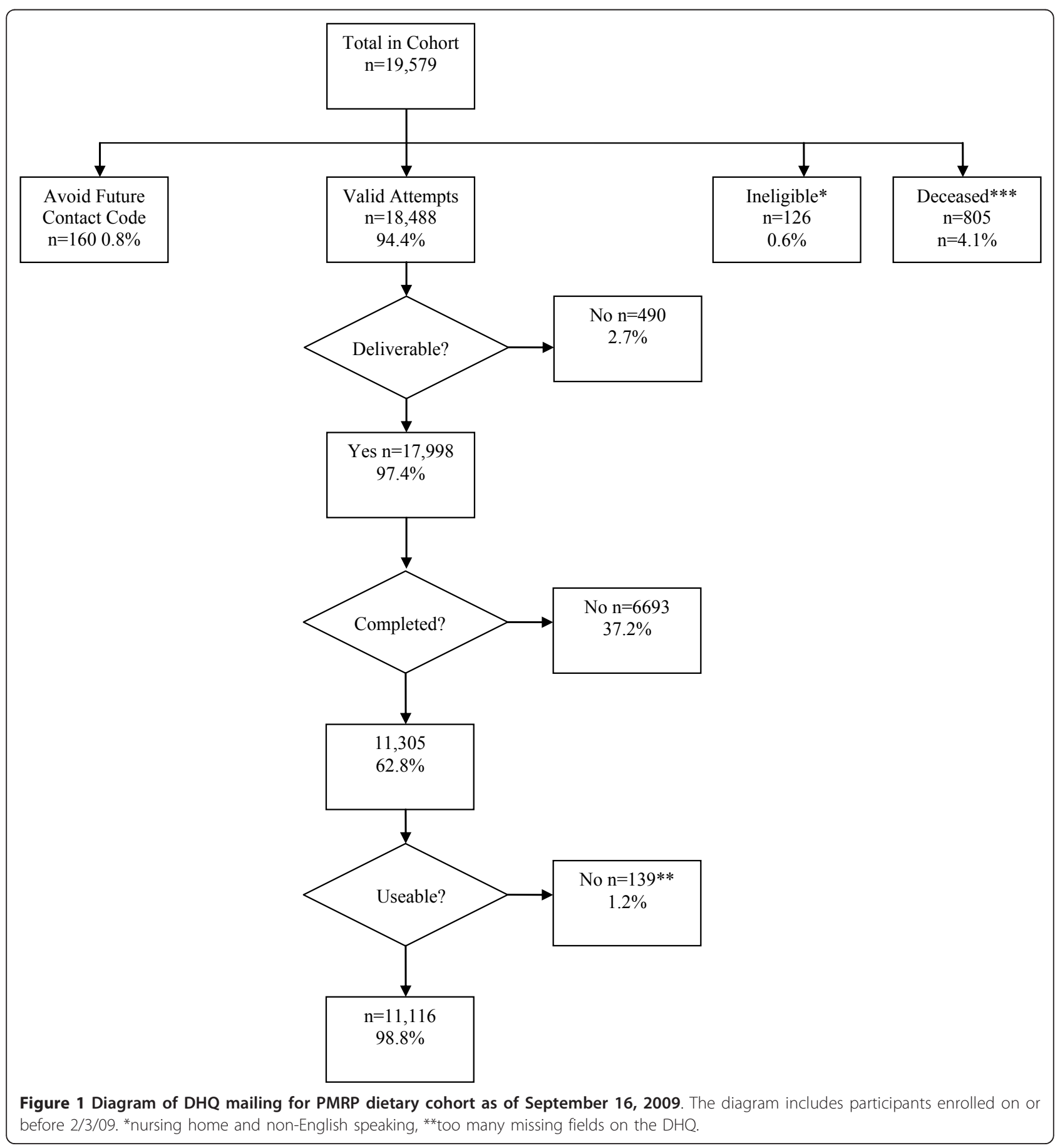


illustrates the tracking of questionnaires mailed out to participants. The 11,166 with dietary information ranged in age from 18 to 98 years (mean 54.9 years) and 6821 (61\%) were female. Demographic characteristics of the dietary cohort and a comparison with non-responders are summarized in Table 1. Responders were more likely to be female, older, and never smokers.
Dietary intake varied by age, gender, smoking and the E4 allele. Trends seen within data are statistically significant, unless otherwise noted. Table 2 compares the percent use of various supplements between females and males of different age groups. The percent use of supplements for females increases as age group increases. Over fifty percent of women in each age group consume

Table 1 Descriptive characteristics of the dietary cohort and comparison with non-responders

\begin{tabular}{|c|c|c|c|c|}
\hline & Males & Females & Overall & Non-responders \\
\hline Subjects (n \%) & $4,34539 \%$ & $6,82161 \%$ & 11,166 & $8,41352 \%$ Female \\
\hline Median age (yr) & 57.1 & 53.4 & 54.9 & 46.4 \\
\hline Minimum & 18 & 18 & 18 & 18 \\
\hline Maximum & 96 & 98 & 98 & 103 \\
\hline Diabetes (n \%) & $76918 \%$ & $90413 \%$ & $167315 \%$ & $125015 \%$ \\
\hline \multicolumn{5}{|l|}{ Smoking history (n \%) } \\
\hline Never & $202447 \%$ & $421162 \%$ & $623556 \%$ & $429851 \%$ \\
\hline Current & $65515 \%$ & $98314 \%$ & $163815 \%$ & $206525 \%$ \\
\hline Other or unknown & $166638 \%$ & $162724 \%$ & $329329 \%$ & $205024 \%$ \\
\hline E4 genotype (n \%) & $119828 \%$ & $175026 \%$ & $294826 \%$ & $227127 \%$ \\
\hline Median BMI (kg/m²) & 28.7 & 27.8 & 28.2 & 27.8 \\
\hline Minimum & 14.7 & 15.0 & 14.7 & 14.9 \\
\hline Maximum & 71.1 & 69.9 & 71.1 & 66.4 \\
\hline Median HDL (mg/dL) & 43.0 & 53.5 & 49.0 & 46.0 \\
\hline Minimum & 16.0 & 15.0 & 15.0 & 11.0 \\
\hline Maximum & 135.0 & 147.0 & 147.0 & 141.0 \\
\hline
\end{tabular}

Table 2 Percent use of supplements by gender and age in the Personalized Medicine Research Project

\begin{tabular}{|c|c|c|c|c|c|c|c|}
\hline Females & & & & Males & & & \\
\hline Age Group & $18-39$ & $40-59$ & $60+$ & Age Group & $18-39$ & $40-59$ & $60+$ \\
\hline \multirow[t]{2}{*}{$\mathrm{N}^{*}$} & 1644 & 2645 & 2512 & $\mathrm{~N}^{*}$ & 824 & 1620 & 1900 \\
\hline & $\%$ use & $\%$ use & $\%$ use & & $\%$ use & $\%$ use & $\%$ use \\
\hline Vit. A IU & 59.6 & 65.1 & 71.2 & Vit. A IU & 38.3 & 47.3 & 56.6 \\
\hline Vit. A RAE & 59.6 & 65.1 & 71.2 & Vit. A RAE & 38.3 & 47.3 & 56.6 \\
\hline Beta Car. & 59.0 & 64.7 & 70.7 & Beta Car. & 37.9 & 47.1 & 56.6 \\
\hline Vit. E & 60.3 & 68.9 & 75.6 & Vit. E & 38.8 & 49.4 & 60.4 \\
\hline Vit. C & 65.2 & 72.5 & 76.6 & Vit. C & 44.7 & 53.4 & 62.5 \\
\hline Thiamin & 59.5 & 66.6 & 71.6 & Thiamin & 38.0 & 47.7 & 57.4 \\
\hline Riboflavin & 59.5 & 66.6 & 71.6 & Riboflavin & 38.0 & 47.7 & 57.4 \\
\hline Niacin & 59.7 & 67.0 & 72.1 & Niacin & 38.2 & 48.3 & 58.6 \\
\hline Vit. B6 & 59.8 & 67.4 & 72.5 & Vit. B6 & 38.3 & 48.0 & 58.3 \\
\hline Folic Acid & 60.2 & 65.3 & 71.7 & Folic Acid & 38.1 & 47.3 & 57.9 \\
\hline Vit. B12 & 58.7 & 64.2 & 70.0 & Vit. B12 & 37.6 & 46.7 & 56.1 \\
\hline Calcium & 27.8 & 55.0 & 69.7 & Calcium & 13.5 & 17.3 & 30.9 \\
\hline Magnesium & 54.1 & 60.0 & 61.1 & Magnesium & 32.9 & 42.3 & 48.8 \\
\hline Iron & 56.5 & 61.9 & 63.4 & Iron & 33.7 & 42.7 & 50.8 \\
\hline Zinc & 54.8 & 61.2 & 62.7 & Zinc & 34.0 & 43.3 & 50.5 \\
\hline Copper & 54.1 & 60.0 & 61.1 & Copper & 32.9 & 42.3 & 48.8 \\
\hline Vit. D & 58.7 & 64.2 & 70.0 & Vit. D & 37.6 & 46.7 & 56.1 \\
\hline Selenium & 0.5 & 1.6 & 2.1 & Selenium & 0.2 & 2.2 & 5.1 \\
\hline
\end{tabular}

${ }^{*} \mathrm{~N}$ refers to the total number of females or males within the particular age group. 
supplements; similar trends are seen for males. When comparing males and females in the same age group, the percent-use of the various supplements is lower in males than in females. Vitamin C supplements are consumed most frequently by both females and males.

Tables 3 and 4 compare the dietary intake between different age groups of females (Tables 3 ) and males (Table 4). Supplement-use summaries for the subset who use supplements are listed at the bottom of each table. The results suggest that with increasing age in females, food energy, total fat, cholesterol, protein, and alcohol intake decreases. Conversely, as women age, the average supplement intake increased. Similar trends were observed in males, regarding the mean and median intake of food energy, total fat, cholesterol, protein, alcohol, and supplement intake.

Tables 5, 6 and 7 illustrate supplement use stratified by age group, gender, smoking, and E4 allele status. Comparing females on smoking status alone, the nonsmokers generally consume more dietary supplements throughout all age groups. This similar trend is seen in males as well. The data show, once again, that females had more supplement use percentages than males when comparing relative age groups. Differences in supplement use are seen between those that have the E4 allele and those that do not. In the nonsmoking females, those with the E4 allele had higher supplement intake than nonsmokers without E4. Current smoking females with E4 have a higher

Table 3 Dietary intake in females by age in the Personalized Medicine Research Project

\begin{tabular}{|c|c|c|c|c|c|c|c|c|c|c|}
\hline \multirow[t]{2}{*}{ Age Group } & \multicolumn{5}{|c|}{$18-39$} & \multirow{2}{*}{$\begin{array}{r}\text { 40-59 } \\
\text { Median }\end{array}$} & \multicolumn{4}{|c|}{$60+$} \\
\hline & $\mathrm{N}^{*}$ & Mean & Median & $\mathrm{N}^{*}$ & Mean & & $\mathrm{N}^{*}$ & Mean & Median & K-W Test $\mathrm{p}$-value \\
\hline Food energy (kcal) & 2147 & 1859.5 & 1637.4 & 2752 & 1674.7 & 1522.7 & 1997 & 1415.5 & 1341.1 & $<0.001$ \\
\hline Total fat (g) & 2147 & 65.2 & 56.1 & 2752 & 61.6 & 53.8 & 1997 & 51.3 & 46.0 & $<0.001$ \\
\hline Cholesterol (mg) & 2147 & 199.3 & 171.4 & 2752 & 185.0 & 161.7 & 1997 & 154.3 & 137.3 & $<0.001$ \\
\hline Protein (g) & 2147 & 71.6 & 64.2 & 2752 & 67.3 & 61.7 & 1997 & 54.5 & 50.8 & $<0.001$ \\
\hline Alcohol (g) & 2147 & 6.6 & 2.1 & 2752 & 5.9 & 1.4 & 1997 & 3.1 & 0.6 & $<0.001$ \\
\hline Vitamin A (IU CSFII) & 2147 & 8958.8 & 6375.6 & 2752 & 9921.8 & 7354.3 & 1997 & 8549.4 & 6695.0 & $<0.001$ \\
\hline Vitamin A (mcg RE CSFII) & 2147 & 1264.8 & 1003.5 & 2752 & 1324.5 & 1096.7 & 1997 & 1157.6 & 999.3 & $<0.001$ \\
\hline Vitamin E (mg ATE CSFII) & 2147 & 8.3 & 6.9 & 2752 & 8.4 & 7.3 & 1997 & 7.5 & 6.6 & $<0.001$ \\
\hline Vitamin C (mg) & 2147 & 134.6 & 101.3 & 2752 & 127.1 & 104.3 & 1997 & 124.5 & 108.2 & $<0.065$ \\
\hline Zinc (mg) & 2147 & 11.0 & 9.8 & 2752 & 10.4 & 9.5 & 1997 & 8.7 & 8.1 & $<0.001$ \\
\hline Selenium (mcg) & 2147 & 86.2 & 76.4 & 2752 & 81.2 & 74.0 & 1997 & 67.0 & 62.1 & $<0.001$ \\
\hline Total Vitamin A Activity (mcg) & 2147 & 838.4 & 719.7 & 2752 & 828.2 & 719.9 & 1997 & 721.8 & 659.8 & $<0.001$ \\
\hline Beta Carotene & 2147 & 3198.6 & 2055.7 & 2752 & 3657.3 & 2524.0 & 1997 & 3101.7 & 2276.5 & $<0.001$ \\
\hline Lutein|Zeaxanthin (mcg NDS) & 2147 & 2444.7 & 1643.3 & 2752 & 2467.9 & 1727.9 & 1997 & 1998.6 & 1474.5 & $<0.001$ \\
\hline Lycopene (mcg NDS R) & 2147 & 7726.0 & 5611.2 & 2752 & 6815.3 & 4998.7 & 1997 & 6096.7 & 4022.5 & $<0.001$ \\
\hline Beta Carotene Equivalents (mcg) & 2147 & 3701.3 & 2373.0 & 2752 & 4237.7 & 2899.1 & 1997 & 3599.2 & 2632.4 & $<0.001$ \\
\hline Vitamin E Total & 2147 & 7.8 & 6.4 & 2752 & 7.9 & 6.7 & 1997 & 6.7 & 5.9 & $<0.001$ \\
\hline Supp. Vitamin A (IU) & 2147 & 2094.6 & 331.8 & 2752 & 2928.0 & 3571.4 & 1997 & 3496.9 & 5000.0 & $<0.001$ \\
\hline Supp. Vitamin A (mcg RAE) & 2147 & 628.4 & 99.6 & 2752 & 878.4 & 1071.4 & 1997 & 1049.1 & 1500.0 & $<0.001$ \\
\hline Supp. Beta Carotene & 2147 & 246.8 & 39.8 & 2752 & 339.6 & 428.6 & 1997 & 410.7 & 600.0 & $<0.001$ \\
\hline Supp. Vitamin E & 2147 & 16.8 & 4.4 & 2752 & 58.5 & 20.1 & 1997 & 78.0 & 20.1 & $<0.001$ \\
\hline Supp. Vitamin C & 2147 & 81.1 & 17.1 & 2752 & 150.7 & 60.0 & 1997 & 176.8 & 60.0 & $<0.001$ \\
\hline Supp. Zinc & 2147 & 6.1 & 1.0 & 2752 & 8.6 & 10.7 & 1997 & 9.2 & 15.0 & $<0.001$ \\
\hline Supp. Selenium & 2147 & 0.3 & 0.0 & 2752 & 0.8 & 0.0 & 1997 & 0.8 & 0.0 & $<0.001$ \\
\hline \multicolumn{11}{|l|}{ Supplement Users Only } \\
\hline Supp. Vitamin A (IU) & 992 & 3497.2 & 5000.0 & 1722 & 4182.1 & 5000.0 & 1789 & 4843.7 & 5000.0 & $<0.001$ \\
\hline Supp. Vitamin A (mcg RAE) & 992 & 1049.2 & 1500.0 & 1722 & 1254.6 & 1500.0 & 1789 & 1453.1 & 1500.0 & $<0.001$ \\
\hline Supp. Beta Carotene & 982 & 410.2 & 600.0 & 1712 & 492.9 & 600.0 & 1775 & 572.1 & 600.0 & $<0.001$ \\
\hline Supp. Vitamin E & 1003 & 24.6 & 20.1 & 1823 & 74.1 & 20.1 & 1900 & 100.0 & 20.1 & $<0.001$ \\
\hline Supp. Vitamin C & 1085 & 115.5 & 60.0 & 1917 & 188.4 & 60.0 & 1924 & 229.1 & 60.0 & $<0.001$ \\
\hline Supp. Zinc (mg) & 900 & 1.4 & 2.0 & 1587 & 1.6 & 2.0 & 1535 & 1.8 & 2.0 & $<0.001$ \\
\hline Supp. Selenium (mcg) & 9 & 42.9 & 42.9 & 43 & 42.9 & 42.9 & 53 & 42.9 & 42.9 & 1.000 \\
\hline
\end{tabular}

${ }^{*} \mathrm{~N}$ refers to the total number of participants per age group. 
Table 4 Dietary intake in males by age in the Personalized Medicine Research Project

\begin{tabular}{|c|c|c|c|c|c|c|c|c|c|c|}
\hline \multirow[t]{2}{*}{ Age Group } & \multicolumn{3}{|c|}{$18-39$} & \multicolumn{3}{|c|}{$40-59$} & \multicolumn{3}{|c|}{$60+$} & \multirow[b]{2}{*}{ K-W Test $\mathrm{p}$-value } \\
\hline & $\mathrm{N}^{*}$ & Mean & Median & $\mathrm{N}^{*}$ & Mean & Median & $\mathrm{N}^{*}$ & Mean & Median & \\
\hline Total fat (g) & 1105 & 99.2 & 85.9 & 1764 & 87.0 & 74.7 & 1515 & 69.6 & 61.9 & $<0.001$ \\
\hline Cholesterol (mg) & 1105 & 304.0 & 258.1 & 1764 & 263.1 & 226.1 & 1515 & 214.9 & 186.8 & $<0.001$ \\
\hline Protein (g) & 1105 & 106.0 & 91.1 & 1764 & 91.7 & 81.3 & 1515 & 71.2 & 64.4 & $<0.001$ \\
\hline Alcohol (g) & 1105 & 21.4 & 5.1 & 1764 & 15.5 & 3.5 & 1515 & 10.2 & 1.8 & $<0.001$ \\
\hline Vitamin A (IU CSFII) & 1105 & 9292.7 & 6715.6 & 1764 & 9490.8 & 7456.3 & 1515 & 8731.1 & 6842.3 & $<0.001$ \\
\hline Vitamin A (mcg RE CSFII) & 1105 & 1442.2 & 1152.8 & 1764 & 1384.7 & 1201.5 & 1515 & 1270.6 & 1086.9 & $<0.001$ \\
\hline Vitamin E (mg ATE CSFII) & 1105 & 10.9 & 8.8 & 1764 & 10.2 & 8.7 & 1515 & 9.0 & 7.8 & $<0.001$ \\
\hline Vitamin C (mg) & 1105 & 157.3 & 113.0 & 1764 & 137.4 & 111.4 & 1515 & 125.8 & 107.5 & $<0.034$ \\
\hline Zinc (mg) & 1105 & 16.3 & 14.0 & 1764 & 14.3 & 12.6 & 1515 & 11.6 & 10.3 & $<0.001$ \\
\hline Selenium (mcg) & 1105 & 130.9 & 114.0 & 1764 & 116.1 & 102.4 & 1515 & 92.1 & 83.5 & $<0.001$ \\
\hline Total Vitamin A Activity (mcg) & 1105 & 1048.6 & 862.2 & 1764 & 944.1 & 838.1 & 1515 & 848.5 & 733.7 & $<0.001$ \\
\hline Beta Carotene & 1105 & 3111.2 & 1971.9 & 1764 & 3290.3 & 2335.0 & 1515 & 3035.9 & 2195.7 & $<0.001$ \\
\hline Lutein|Zeaxanthin (mcg NDS) & 1105 & 2640.6 & 1740.4 & 1764 & 2368.8 & 1773.8 & 1515 & 2140.5 & 1544.8 & $<0.001$ \\
\hline Lycopene (mcg NDS R) & 1105 & 10491 & 7941.1 & 1764 & 9438.5 & 6621.3 & 1515 & 7770.7 & 5163.8 & $<0.001$ \\
\hline Beta Carotene Equivalents (mcg) & 1105 & 3571.6 & 2266.4 & 1764 & 3818.0 & 2697.1 & 1515 & 3521.6 & 2538.3 & $<0.001$ \\
\hline Vitamin E Total & 1105 & 10.0 & 8.1 & 1764 & 9.3 & 8.0 & 1515 & 7.9 & 6.8 & $<0.001$ \\
\hline Supp. Vitamin A (IU) & 1105 & 1222.9 & 0.0 & 1764 & 2258.9 & 82.1 & 1515 & 2806.4 & 3571.4 & $<0.001$ \\
\hline Supp. Vitamin A (mcg RAE) & 1105 & 366.9 & 0.0 & 1764 & 677.7 & 24.6 & 1515 & 841.9 & 1071.4 & $<0.001$ \\
\hline Supp. Beta Carotene & 1105 & 144.1 & 0.0 & 1764 & 264.9 & 9.9 & 1515 & 327.5 & 428.6 & $<0.001$ \\
\hline Supp. Vitamin E & 1105 & 11.6 & 0.0 & 1764 & 35.9 & 2.4 & 1515 & 50.5 & 20.1 & $<0.001$ \\
\hline Supp. Vitamin C & 1105 & 54.8 & 0.0 & 1764 & 106.3 & 17.1 & 1515 & 130.8 & 60.0 & $<0.001$ \\
\hline Supp. Zinc & 1105 & 3.8 & 0.0 & 1764 & 6.6 & 0.0 & 1515 & 7.8 & 0.0 & $<0.001$ \\
\hline Supp. Selenium & 1105 & 0.2 & 0.0 & 1764 & 1.2 & 0.0 & 1515 & 2.2 & 0.0 & $<0.001$ \\
\hline \multicolumn{11}{|l|}{ Supplement Users Only } \\
\hline Supp. Vitamin A (IU) & 316 & 3059.8 & 3571.4 & 766 & 4229.3 & 5000.0 & 1075 & 4930.3 & 5000.0 & $<0.001$ \\
\hline Supp. Vitamin A (mcg RAE) & 316 & 917.9 & 1071.4 & 766 & 1268.8 & 1500.0 & 1075 & 1479.1 & 1500.0 & $<0.001$ \\
\hline Supp. Beta Carotene & 312 & 360.5 & 428.6 & 763 & 494.1 & 600.0 & 1076 & 579.4 & 600.0 & $<0.001$ \\
\hline Supp. Vitamin E & 320 & 22.9 & 14.4 & 801 & 61.6 & 20.1 & 1147 & 83.4 & 20.1 & $<0.001$ \\
\hline Supp. Vitamin C & 368 & 115.6 & 60.0 & 865 & 173.2 & 60.0 & 1187 & 212.1 & 60.0 & $<0.001$ \\
\hline Supp. Zinc (mg) & 271 & 1.2 & 1.4 & 685 & 1.6 & 2.0 & 927 & 1.9 & 2.0 & $<0.001$ \\
\hline Supp. Selenium (mcg) & 2 & 42.9 & 42.9 & 36 & 42.9 & 42.9 & 96 & 42.9 & 42.9 & 1.000 \\
\hline
\end{tabular}

${ }^{*} \mathrm{~N}$ refers to the total number of participants per age group.

supplement intake than those without E4. This trend is relatively consistent throughout all age groups in females. The data show some inconsistencies between males and females. In nonsmoking males between ages 18-39, those with E4 have higher percent use than those without E4; in the same age group, smokers without the E4 allele had higher use of supplements. In the other two age groups, nonsmoking males with $\mathrm{E} 4$ have lower percent use of supplements than those without E4. Current smoking males with E4 had a lower percent use than those without E4. This trend is seen in current smokers among each age group in males. Supplement use by E4 differed between genders. In general, females with E4 had higher supplement use percentages, and males with E4 had lower supplement use percentages compared to those without E4.

Table 8 compares the dietary intake between smoking and nonsmoking females and males. For females, the data suggest that the dietary intake for food energy, total fat, cholesterol, alcohol, vitamin E (mg ATE CSFII), selenium, and lycopene was higher in smokers versus nonsmokers. Furthermore, the dietary intake for vitamin A (IU CSFII), vitamin A (mcg RE CSFII), and vitamin $C$ was higher in nonsmokers than in smokers. No differences were seen in supplement intake between the two groups. Differences can also be seen between smokers and nonsmokers when comparing $25 \%$ and $75 \%$ quartile values. The results regarding smoking and dietary intake for males were not statistically significant. Nonsmokers generally consumed healthier diets, as evidenced by using more supplements, consuming higher dietary vitamin $\mathrm{C}$, and consuming less alcohol.

Tables 8 and 9 illustrate the supplement intake between females (Table 9) and males (Table 10) 
Table 5 Percent use of supplements by gender, smoking status, and E4 in subjects aged 18-39 in the Personalized Medicine Research Project

\begin{tabular}{|c|c|c|c|c|}
\hline & \multicolumn{2}{|c|}{ Never Smoked } & \multicolumn{2}{|c|}{ Current Smokers } \\
\hline & With E4 & No E4 & With E4 & No E4 \\
\hline \multicolumn{5}{|l|}{ Females } \\
\hline \multirow[t]{2}{*}{$N^{*}$} & 293 & 775 & 104 & 296 \\
\hline & $\%$ Use & $\%$ Use & $\%$ Use & $\%$ Use \\
\hline Vit. A IU & 68.9 & 61.4 & 49.0 & 48.0 \\
\hline Vit. A RAE & 68.9 & 61.4 & 49.0 & 48.0 \\
\hline Beta Car. & 68.3 & 60.5 & 49.0 & 48.3 \\
\hline Vit. E & 69.6 & 61.9 & 51.0 & 49.0 \\
\hline Vit. C & 73.0 & 66.7 & 52.9 & 53.7 \\
\hline Thiamin & 68.3 & 60.9 & 49.0 & 48.6 \\
\hline Riboflavin & 68.3 & 60.9 & 49.0 & 48.6 \\
\hline Niacin & 68.3 & 61.4 & 49.0 & 48.6 \\
\hline Vit. B6 & 68.3 & 61.4 & 49.0 & 49.0 \\
\hline Folic Acid & 67.9 & 62.1 & 50.0 & 49.0 \\
\hline Vit. B12 & 67.9 & 60.1 & 48.1 & 48.0 \\
\hline Calcium & 32.4 & 25.0 & 27.9 & 25.0 \\
\hline Magnesium & 62.1 & 55.4 & 44.2 & 44.9 \\
\hline Iron & 63.1 & 57.5 & 45.2 & 49.0 \\
\hline Zinc & 63.1 & 56.0 & 45.2 & 45.6 \\
\hline Copper & 62.1 & 55.4 & 44.2 & 44.9 \\
\hline Vit. D & 67.9 & 60.1 & 48.1 & 48.0 \\
\hline Selenium & 0.0 & 1.0 & 0.0 & 0.3 \\
\hline \multicolumn{5}{|l|}{ Males } \\
\hline \multirow[t]{2}{*}{$N^{*}$} & 125 & 377 & 59 & 156 \\
\hline & $\%$ Use & \% Use & $\%$ Use & $\%$ Use \\
\hline Vit. A IU & 40.8 & 39.5 & 30.5 & 35.9 \\
\hline Vit. A RAE & 40.8 & 39.5 & 30.5 & 35.9 \\
\hline Beta Car. & 42.4 & 38.7 & 28.8 & 35.3 \\
\hline Vit. E & 40.8 & 39.5 & 33.9 & 37.2 \\
\hline Vit. C & 46.4 & 43.8 & 40.7 & 46.8 \\
\hline Thiamin & 40.8 & 38.7 & 30.5 & 35.9 \\
\hline Riboflavin & 40.8 & 38.7 & 30.5 & 35.9 \\
\hline Niacin & 41.6 & 38.7 & 32.2 & 35.9 \\
\hline Vit. B6 & 40.8 & 38.7 & 32.2 & 36.5 \\
\hline Folic Acid & 40.8 & 38.7 & 32.2 & 35.9 \\
\hline Vit. B12 & 40.8 & 38.7 & 28.8 & 35.3 \\
\hline Calcium & 16.0 & 11.9 & 15.3 & 12.8 \\
\hline Magnesium & 34.4 & 33.7 & 25.4 & 30.8 \\
\hline Iron & 34.4 & 33.7 & 30.5 & 33.3 \\
\hline Zinc & 35.2 & 34.7 & 30.5 & 31.4 \\
\hline Copper & 34.4 & 33.7 & 25.4 & 30.8 \\
\hline Vit. D & 40.8 & 38.7 & 28.8 & 35.3 \\
\hline Selenium & 0.0 & 0.30 & 1.70 & 0.0 \\
\hline
\end{tabular}

${ }^{*} \mathrm{~N}$ refers to the number of participants.
Table 6 Percent use of supplements by gender, smoking status, and E4 in subjects aged 40-59 in the Personalized Medicine Research Project

\begin{tabular}{|c|c|c|c|c|}
\hline & \multicolumn{2}{|c|}{ Never Smoked } & \multicolumn{2}{|c|}{ Current Smokers } \\
\hline & With E4 & No E4 & With E4 & No E4 \\
\hline \multicolumn{5}{|l|}{ Females } \\
\hline \multirow[t]{2}{*}{$N^{*}$} & 426 & 1186 & 105 & 306 \\
\hline & $\%$ Use & $\%$ Use & $\%$ Use & $\%$ Use \\
\hline Vit. A IU & 70.9 & 64.3 & 65.7 & 57.8 \\
\hline Vit. A RAE & 70.9 & 64.3 & 65.7 & 57.8 \\
\hline Beta Car. & 70.4 & 63.8 & 65.7 & 56.9 \\
\hline Vit. E & 74.2 & 68.0 & 65.7 & 61.1 \\
\hline Vit. C & 78.4 & 72.2 & 71.4 & 64.1 \\
\hline Thiamin & 73.5 & 65.5 & 67.6 & 58.5 \\
\hline Riboflavin & 73.5 & 65.5 & 67.6 & 58.5 \\
\hline Niacin & 73.9 & 65.9 & 67.6 & 58.5 \\
\hline Vit. B6 & 73.5 & 66.6 & 69.5 & 59.5 \\
\hline Folic Acid & 71.4 & 64.6 & 67.6 & 57.5 \\
\hline Vit. B12 & 70.2 & 63.4 & 65.7 & 56.9 \\
\hline Calcium & 59.9 & 55.6 & 42.9 & 43.8 \\
\hline Magnesium & 67.1 & 58.9 & 56.2 & 53.6 \\
\hline Iron & 68.3 & 61.4 & 57.1 & 54.9 \\
\hline Zinc & 67.8 & 59.9 & 57.1 & 54.2 \\
\hline Copper & 67.1 & 58.9 & 56.2 & 53.6 \\
\hline Vit. D & 70.2 & 63.4 & 65.7 & 56.9 \\
\hline Selenium & 1.9 & 1.5 & 1.9 & 1.0 \\
\hline \multicolumn{5}{|l|}{ Males } \\
\hline \multirow[t]{2}{*}{$N^{*}$} & 230 & 624 & 91 & 215 \\
\hline & $\%$ Use & $\%$ Use & $\%$ Use & $\%$ Use \\
\hline Vit. A IU & 46.1 & 47.1 & 41.8 & 46.0 \\
\hline Vit. A RAE & 46.1 & 47.1 & 41.8 & 46.0 \\
\hline Beta Car. & 46.1 & 47.1 & 38.5 & 46.5 \\
\hline Vit. E & 47.8 & 49.5 & 40.7 & 47.4 \\
\hline Vit. C & 50.9 & 53.0 & 49.5 & 51.6 \\
\hline Thiamin & 45.7 & 47.6 & 38.5 & 47.0 \\
\hline Riboflavin & 45.7 & 47.6 & 38.5 & 47.0 \\
\hline Niacin & 45.7 & 48.2 & 38.5 & 47.9 \\
\hline Vit. B6 & 46.5 & 47.6 & 39.6 & 47.0 \\
\hline Folic Acid & 46.5 & 47.1 & 38.5 & 46.5 \\
\hline Vit. B12 & 45.7 & 46.8 & 37.4 & 46.0 \\
\hline Calcium & 10.4 & 17.8 & 13.2 & 15.3 \\
\hline Magnesium & 43.0 & 41.5 & 33.0 & 41.9 \\
\hline Iron & 43.0 & 42.0 & 34.1 & 41.9 \\
\hline Zinc & 43.9 & 42.1 & 35.2 & 42.8 \\
\hline Copper & 43.0 & 41.5 & 33.0 & 41.9 \\
\hline Vit. D & 45.7 & 46.8 & 37.4 & 46.0 \\
\hline Selenium & 2.2 & 1.4 & 2.2 & 2.8 \\
\hline
\end{tabular}

${ }^{*} \mathrm{~N}$ refers to the number of participants. 
Table 7 Percent use of supplements by gender, smoking status, and E4 in subjects aged 60 and older in the Personalized Medicine Research Project

\begin{tabular}{|c|c|c|c|c|}
\hline & \multicolumn{2}{|c|}{ Never Smoked } & \multicolumn{2}{|c|}{ Current Smokers } \\
\hline & With E4 & No E4 & With E4 & No E4 \\
\hline \multicolumn{5}{|l|}{ Females } \\
\hline \multirow[t]{2}{*}{$N^{*}$} & 374 & 1157 & 39 & 133 \\
\hline & $\%$ Use & $\%$ Use & $\%$ Use & $\%$ Use \\
\hline Vit. A IU & 73.3 & 71.3 & 71.8 & 60.9 \\
\hline Vit. A RAE & 73.3 & 71.3 & 71.8 & 60.9 \\
\hline Beta Car. & 72.7 & 70.7 & 71.8 & 61.7 \\
\hline Vit. E & 78.3 & 76.2 & 74.4 & 66.9 \\
\hline Vit. C & 79.9 & 76.1 & 74.4 & 72.2 \\
\hline Thiamin & 74.3 & 71.6 & 71.8 & 61.7 \\
\hline Riboflavin & 74.3 & 71.6 & 71.8 & 61.7 \\
\hline Niacin & 74.3 & 72.4 & 71.8 & 61.7 \\
\hline Vit. B6 & 74.9 & 72.5 & 71.8 & 62.4 \\
\hline Folic Acid & 74.1 & 71.3 & 71.8 & 63.9 \\
\hline Vit. B12 & 72.5 & 69.8 & 71.8 & 60.2 \\
\hline Calcium & 68.2 & 72.0 & 56.4 & 58.6 \\
\hline Magnesium & 64.2 & 60.3 & 64.1 & 51.1 \\
\hline Iron & 65.8 & 62.9 & 64.1 & 54.1 \\
\hline Zinc & 65.0 & 62.1 & 66.7 & 51.9 \\
\hline Copper & 64.2 & 60.3 & 64.1 & 51.1 \\
\hline Vit. D & 72.5 & 69.8 & 71.8 & 60.2 \\
\hline Selenium & 1.6 & 2.0 & 0.0 & 3.8 \\
\hline \multicolumn{5}{|l|}{ Males } \\
\hline \multirow[t]{2}{*}{$\mathrm{N}^{*}$} & 190 & 477 & 33 & 101 \\
\hline & $\%$ Use & $\%$ Use & $\%$ Use & $\%$ Use \\
\hline Vit. A IU & 47.9 & 57.9 & 39.4 & 56.4 \\
\hline Vit. A RAE & 47.9 & 57.9 & 39.4 & 56.4 \\
\hline Beta Car. & 47.9 & 58.1 & 39.4 & 57.4 \\
\hline Vit. E & 53.7 & 60.6 & 45.5 & 57.4 \\
\hline Vit. C & 54.2 & 63.5 & 48.5 & 61.4 \\
\hline Thiamin & 48.4 & 59.5 & 39.4 & 56.4 \\
\hline Riboflavin & 48.4 & 59.5 & 39.4 & 56.4 \\
\hline Niacin & 51.1 & 60.4 & 39.4 & 57.4 \\
\hline Vit. B6 & 48.4 & 60.6 & 45.5 & 57.4 \\
\hline Folic Acid & 48.4 & 58.7 & 45.5 & 57.4 \\
\hline Vit. B12 & 47.4 & 57.2 & 39.4 & 56.4 \\
\hline Calcium & 26.8 & 32.1 & 33.3 & 29.7 \\
\hline Magnesium & 40.5 & 50.1 & 36.4 & 48.5 \\
\hline Iron & 42.1 & 52.4 & 36.4 & 50.5 \\
\hline Zinc & 42.1 & 52.0 & 39.4 & 49.5 \\
\hline Copper & 40.5 & 50.1 & 36.4 & 48.5 \\
\hline Vit. D & 47.4 & 57.2 & 39.4 & 56.4 \\
\hline Selenium & 4.7 & 6.5 & 3.0 & 1.0 \\
\hline
\end{tabular}

*N refers to the number of participants.

stratified by having the E4 allele or not. The data suggest that females with the E4 allele have higher supplement intake than those without it; however, when looking at the "supplement users only" data, there is little to no difference by E4 status. As for males, the data suggest that those without the E4 allele have higher supplement intake. With some exceptions, the same general trend is seen within the "supplement users only" data.

\section{Discussion}

The dietary intake of participants in the Personalized Medicine Research Project (PMRP) is a useful resource to assist in studies regarding gene-diet interactions. Statistically significant findings were seen when analyzing the PMRP dietary data for differences associated with smoking, alcohol consumption, and the APOE genotype.

The National Health and Nutrition Examination Survey (NHANES) is a survey that documents dietary intake on a yearly basis. Comparing the PMRP dietary intake of macronutrients with that of NHANES, the PMRP dietary intake is relatively similar. In ages eighteen and above, percent energy from protein, carbohydrates, total fat, and saturated fat are similar between the PMRP and NHANES. NHANES data revealed slightly higher food energy, cholesterol, natural folate, and sodium intake. PMRP intake was significantly higher for calcium [18]. This finding could be due to the higher consumption of dairy foods and vegetables associated the farming in Wisconsin.

Differences have been seen in dietary intake between smokers and nonsmokers. Interactions between diet and smoking can lead to negative health outcomes. Findings of previous studies suggest that smokers consume less fiber, vegetables, whole grains, fruits but more bacon/ luncheon meats, whole milk, and calories in general [3]. Smokers also are less likely than nonsmokers to consume vitamins, minerals and/or supplements [3]. Our results are generally consistent with previous findings. In PMRP, women who smoke have a lower intake of supplements and vitamins, and a higher intake in food energy, fat, cholesterol, and protein. Similarly, supplement intake was lower and alcohol consumption was higher in smoking males.

Studies have shown the APOE gene to be associated with increased risk for coronary heart disease (CHD) and Alzheimer's Disease. Smoking increases the risk for $\mathrm{CHD}$ alone, but its interaction with the APOE4 genotype can cause an even higher risk [2]. This demonstrates a possible gene-environment interaction. Our findings suggest that females with the $\mathrm{E} 4$ allele have higher supplement intake and smokers with the E4 allele have slightly lower use. Males with the E4 allele have lower supplement intake, but higher use is seen in nonsmokers. These data suggest that people may have started supplement use to prevent diseases for which they have in increased risk (possibly due to family history) and these diseases are associated with APOE. Vitamin E supplementation has been shown to decrease the 
Table 8 Dietary intake by gender and smoking status in the Personalized Medicine Research Project. The total number of participants who smoked and never smoked is indicated by " $\mathrm{N}$ " beneath the respective category

\begin{tabular}{|c|c|c|c|c|c|c|c|}
\hline \multicolumn{8}{|l|}{ Females } \\
\hline & \multicolumn{3}{|c|}{ Never Smoked } & \multicolumn{3}{|c|}{ Smoked $100+$} & \\
\hline \multirow[t]{2}{*}{$\mathrm{N}^{*}$} & & 4262 & & 2538 & & & \\
\hline & $25 \%$ & Median & $75 \%$ & $25 \%$ & Median & $75 \%$ & Wilcoxon p-value \\
\hline Food energy (kcal) & 1092.3 & 1468.8 & 1942.1 & 1146.3 & 1539.9 & 2066.2 & $<0.001$ \\
\hline Total fat (g) & 35.7 & 51.2 & 72.3 & 37.6 & 54.8 & 77.2 & $<0.001$ \\
\hline Cholesterol (mg) & 102.8 & 153.8 & 220.1 & 109.6 & 164.2 & 235.3 & $<0.001$ \\
\hline Protein $(\mathrm{g})$ & 42.4 & 58.6 & 78.3 & 42.9 & 60.0 & 81.1 & 0.056 \\
\hline Alcohol (g) & 0.1 & 1.1 & 3.3 & 0.3 & 1.7 & 6.3 & $<0.001$ \\
\hline Vitamin A (IU CSFII) & 4394.1 & 6915.6 & 11608 & 4104.1 & 6639.4 & 11198 & 0.002 \\
\hline Vitamin A (mcg RE CSFII) & 703.1 & 1051.3 & 1563.0 & 654.6 & 1005.5 & 1536.2 & 0.003 \\
\hline Vitamin E (mg ATE CSFII) & 4.7 & 6.8 & 9.9 & 4.8 & 7.2 & 10.5 & 0.007 \\
\hline Vitamin C (mg) & 69.7 & 107.1 & 158.2 & 63.2 & 100.4 & 157.4 & $<0.001$ \\
\hline Zinc (mg) & 6.6 & 9.1 & 12.1 & 6.6 & 9.3 & 12.4 & 0.089 \\
\hline Selenium (mcg) & 50.5 & 70.2 & 94.2 & 52.4 & 72.9 & 99.2 & 0.002 \\
\hline Total Vitamin A Activity (mcg) & 479.9 & 706.7 & 989.6 & 451.1 & 692.1 & 1000.1 & 0.206 \\
\hline Beta Carotene & 1374.5 & 2316.2 & 4265.4 & 1267.5 & 2266.6 & 4176.5 & 0.060 \\
\hline Lutein|Zeaxanthin (mcg NDS) & 1029.3 & 1636.6 & 2579.3 & 1002.5 & 1615.4 & 2619.1 & 0.506 \\
\hline Lycopene (mcg NDS R) & 3026.7 & 4815.3 & 7901.4 & 3194.0 & 5075.2 & 8470.1 & 0.003 \\
\hline Beta Carotene Equivalents (mcg) & 1586.3 & 2689.2 & 4912.3 & 1468.6 & 2609.5 & 4793.5 & 0.026 \\
\hline Vitamin E Total & 4.4 & 6.3 & 9.2 & 4.4 & 6.5 & 9.6 & 0.026 \\
\hline \multicolumn{8}{|l|}{ Males } \\
\hline & \multicolumn{3}{|c|}{ Never Smoked } & \multicolumn{3}{|c|}{ Smoked 100+ } & \\
\hline \multirow[t]{2}{*}{$\mathrm{N}^{*}$} & & 2045 & & & 2233 & & \\
\hline & $25 \%$ & Median & $75 \%$ & $25 \%$ & Median & $75 \%$ & Wilcoxon p-value \\
\hline Food energy (kcal) & 1499.7 & 2051.0 & 2772.5 & 1426.6 & 1955.4 & 2720.8 & 0.050 \\
\hline Total fat (g) & 50.4 & 73.9 & 104.4 & 49.2 & 71.2 & 104.9 & 0.156 \\
\hline Cholesterol (mg) & 147.1 & 222.4 & 324.5 & 147.5 & 216.6 & 326.0 & 0.685 \\
\hline Protein (g) & 57.6 & 79.3 & 110.3 & 54.1 & 75.5 & 105.4 & 0.001 \\
\hline Alcohol (g) & 0.6 & 2.8 & 10.0 & 0.6 & 3.5 & 15.2 & 0.001 \\
\hline Vitamin A (IU CSFII) & 4734.1 & 7164.8 & 11634 & 4505.0 & 6892.0 & 10910 & 0.054 \\
\hline Vitamin A (mcg RE CSFII) & 777.6 & 1179.8 & 1760.9 & 744.9 & 1109.3 & 1652.2 & 0.002 \\
\hline Vitamin E (mg ATE CSFII) & 5.8 & 8.4 & 12.1 & 5.7 & 8.3 & 12.2 & 0.342 \\
\hline Vitamin C (mg) & 73.1 & 113.1 & 176.0 & 67.8 & 107.5 & 167.6 & 0.004 \\
\hline Zinc (mg) & 8.9 & 12.2 & 17.4 & 8.5 & 11.8 & 16.7 & 0.006 \\
\hline Selenium (mcg) & 71.3 & 98.8 & 136.8 & 68.2 & 96.8 & 134.2 & 0.148 \\
\hline Total Vitamin A Activity (mcg) & 554.6 & 834.8 & 1209.9 & 523.7 & 785.4 & 1154.5 & 0.001 \\
\hline Beta Carotene & 1294.6 & 2163.3 & 3954.2 & 1333.7 & 2184.9 & 3822.1 & 0.813 \\
\hline Lutein|Zeaxanthin (mcg NDS) & 1103.4 & 1737.5 & 2696.4 & 1030.2 & 1633.7 & 2641.8 & 0.072 \\
\hline Lycopene (mcg NDS R) & 3976.0 & 6436.8 & 10382 & 3846.7 & 6409.3 & 10736 & 0.780 \\
\hline Beta Carotene Equivalents (mcg) & 1499.2 & 2499.0 & 4593.6 & 1528.5 & 2523.0 & 4431.0 & 0.705 \\
\hline Vitamin E Total & 5.4 & 7.6 & 11.1 & 5.2 & 7.5 & 11.1 & 0.196 \\
\hline
\end{tabular}

${ }^{*} \mathrm{~N}$ refers to the number of participants.

risk of some diseases and supplements are marketed directly to consumers for this purpose.

One strength of the PMRP dietary intake data is the size of the cohort that the data includes. The relatively high response rate is another strength of the resource. However, there were some response limitations. For early participants, dietary data were collected several years after their initial enrollment. The initial 17,000 participants were enrolled within the first eighteen months after the project began in 2002. The first set of mailings was not sent until 2006. Approximately $4 \%$ of participants were deceased by the time the DHQs were mailed. $2.7 \%$ of participants were not able to be contacted. Males were less 
Table 9 Supplement intake by APOE4 genotype in females in the Personalized Medicine Research Project

\begin{tabular}{|c|c|c|c|c|c|c|c|c|c|}
\hline \multirow[b]{2}{*}{ Nutrient } & \multicolumn{4}{|c|}{ With E4 } & \multicolumn{4}{|c|}{ No E4 } & \multirow[b]{2}{*}{ Wilcoxon p-value } \\
\hline & $N^{*}$ & Mean & S.D. & Median & $N^{*}$ & Mean & S.D. & Median & \\
\hline Supp. Vitamin A (IU) & 1750 & 2965.2 & 2801.5 & 3571.4 & 5071 & 2789.8 & 2845.7 & 3571.4 & 0.006 \\
\hline Supp. Vitamin A (mcg RAE) & 1750 & 889.6 & 840.4 & 1071.4 & 5071 & 836.9 & 853.7 & 1071.4 & 0.006 \\
\hline Supp. Beta Carotene & 1750 & 350.6 & 329.0 & 428.6 & 5071 & 325.1 & 323.0 & 428.6 & 0.002 \\
\hline Supp. Vitamin E & 1750 & 52.6 & 113.6 & 20.1 & 5071 & 50.8 & 108.8 & 20.1 & 0.046 \\
\hline Supp. Vitamin C & 1750 & 140.5 & 273.5 & 60.0 & 5071 & 134.4 & 262.3 & 60.0 & 0.076 \\
\hline Supp. Thiamin (mg) & 1750 & 1.4 & 2.0 & 1.5 & 5071 & 1.3 & 1.9 & 1.1 & 0.002 \\
\hline Supp. Riboflavin (mg) & 1750 & 1.3 & 1.5 & 1.7 & 5071 & 1.2 & 1.5 & 1.2 & 0.002 \\
\hline Supp. Niacin (mg) & 1750 & 13.7 & 13.4 & 20.0 & 5071 & 12.7 & 12.9 & 14.3 & 0.003 \\
\hline Supp. Vitamin B6 (mg) & 1750 & 4.4 & 10.6 & 2.0 & 5071 & 4.3 & 10.8 & 1.4 & 0.014 \\
\hline Supp. Folic Acid (mcg) & 1750 & 230.2 & 191.8 & 285.7 & 5071 & 215.8 & 194.8 & 285.7 & 0.004 \\
\hline Supp. Vitamin B12 (mcg) & 1750 & 3.3 & 2.8 & 4.3 & 5071 & 3.1 & 2.8 & 4.3 & 0.002 \\
\hline Supp. Vitamin D (mcg) & 1750 & 286.6 & 365.6 & 16.6 & 5071 & 290.5 & 371.6 & 16.6 & 0.866 \\
\hline Supp. Calcium (mg) & 1750 & 50.8 & 47.3 & 71.4 & 5071 & 47.1 & 47.2 & 28.6 & 0.003 \\
\hline Supp. Magnesium (mg) & 1750 & 10.4 & 10.3 & 12.9 & 5071 & 9.9 & 10.2 & 12.9 & 0.111 \\
\hline Supp. Iron (mg) & 1750 & 8.4 & 8.2 & 10.7 & 5071 & 7.9 & 8.3 & 4.3 & 0.007 \\
\hline Supp. Zinc (mg) & 1750 & 1.0 & 0.9 & 1.4 & 5071 & 0.9 & 0.9 & 0.6 & 0.003 \\
\hline Supp. Copper (mg) & 1750 & 221.4 & 186.7 & 285.7 & 5071 & 205.9 & 188.0 & 285.7 & 0.002 \\
\hline Supp. Selenium (mcg) & 1750 & 0.6 & 4.9 & 0.0 & 5071 & 0.7 & 5.4 & 0.0 & 0.375 \\
\hline \multicolumn{10}{|l|}{ Supplement Users Only } \\
\hline Supp. Vitamin A (IU) & 1208 & 4295.6 & 2377.5 & 5000.0 & 3295 & 4293.5 & 2450.7 & 5000.0 & 0.931 \\
\hline Supp. Vitamin A (mcg RAE) & 1208 & 1288.7 & 713.3 & 1500.0 & 3295 & 1288.0 & 735.2 & 1500.0 & 0.931 \\
\hline Supp. Beta Carotene & 1201 & 510.9 & 275.4 & 600.0 & 3268 & 504.4 & 267.2 & 600.0 & 0.558 \\
\hline Supp. Vitamin E & 1261 & 73.1 & 128.1 & 20.1 & 3465 & 74.3 & 124.8 & 20.1 & 0.468 \\
\hline Supp. Vitamin C & 1314 & 187.2 & 301.5 & 60.0 & 3612 & 188.6 & 293.9 & 60.0 & 0.262 \\
\hline Supp. Thiamin (mg) & 1228 & 2.0 & 2.1 & 1.5 & 3323 & 1.9 & 2.1 & 1.5 & 0.841 \\
\hline Supp. Riboflavin (mg) & 1228 & 1.9 & 1.5 & 1.7 & 3323 & 1.8 & 1.4 & 1.7 & 0.841 \\
\hline Supp. Niacin (mg) & 1231 & 19.5 & 11.8 & 20.0 & 3346 & 19.2 & 11.3 & 20.0 & 0.857 \\
\hline Supp. Vitamin B6 (mg) & 1234 & 6.2 & 12.2 & 2.0 & 3366 & 6.5 & 12.7 & 2.0 & 0.634 \\
\hline Supp. Vitamin B12 (mcg) & 1220 & 330.2 & 140.6 & 400.0 & 3310 & 330.6 & 142.0 & 400.0 & 0.954 \\
\hline Supp. Folic Acid (mcg) & 1195 & 4.9 & 2.0 & 6.0 & 3238 & 4.8 & 2.0 & 6.0 & 0.697 \\
\hline Supp. Vitamin D (mcg) & 941 & 533.0 & 342.5 & 500.0 & 2727 & 540.2 & 349.1 & 500.0 & 0.686 \\
\hline Supp. Calcium (mg) & 1086 & 81.9 & 32.5 & 100.0 & 2936 & 81.4 & 32.5 & 100.0 & 0.681 \\
\hline Supp. Magnesium (mg) & 1110 & 16.4 & 8.2 & 18.0 & 3059 & 16.5 & 8.0 & 18.0 & 0.290 \\
\hline Supp. Iron (mg) & 1107 & 13.2 & 6.5 & 15.0 & 2998 & 13.3 & 6.6 & 15.0 & 0.908 \\
\hline Supp. Zinc (mg) & 1086 & 1.6 & 0.6 & 2.0 & 2936 & 1.6 & 0.7 & 2.0 & 0.681 \\
\hline Supp. Copper (mg) & 1195 & 324.2 & 133.1 & 400.0 & 3238 & 322.5 & 133.1 & 400.0 & 0.697 \\
\hline Supp. Selenium (mcg) & 23 & 42.9 & 0.0 & 42.9 & 82 & 42.9 & 0.0 & 42.9 & 1.000 \\
\hline
\end{tabular}

${ }^{*} \mathrm{~N}$ refers to the number of participants.

likely to respond to the questionnaire. Although this information should be considered, the percentages are quite low and do not present a strong impact on the collected data.

\section{Conclusions}

Detailed dietary history data are available for more than 11,000 adult participants in a biobank with DNA, plasma and serum samples linked to a comprehensive electronic health record. The cohort is representative of the population of central Wisconsin. The dietary intake data will be a valuable resource for studies of gene-environment interactions. The Diet History Questionnaire should be followed up with periodic updates to assess changes in intake over time. The PMRP welcomes collaboration to enhance and expand gene-environment research. 
Table 10 Supplement intake by APOE4 genotype in males in the Personalized Medicine Research Project

\begin{tabular}{|c|c|c|c|c|c|c|c|c|c|}
\hline \multirow[b]{2}{*}{ Nutrient } & \multicolumn{4}{|c|}{ With E4 } & \multicolumn{4}{|c|}{ No E4 } & \multirow[b]{2}{*}{ Wilcoxon p-value } \\
\hline & N & Mean & S.D. & Median & $N$ & Mean & S.D. & Median & \\
\hline Supp. Vitamin A (IU) & 1198 & 2068.6 & 2769.4 & 0.0 & 3146 & 2234.1 & 2781.4 & 82.1 & 0.047 \\
\hline Supp. Vitamin A (mcg RAE) & 1198 & 620.6 & 830.8 & 0.0 & 3146 & 670.2 & 834.4 & 24.6 & 0.047 \\
\hline Supp. Beta Carotene & 1198 & 239.8 & 301.6 & 0.0 & 3146 & 262.4 & 322.6 & 9.9 & 0.043 \\
\hline Supp. Vitamin E & 1198 & 34.0 & 92.0 & 0.3 & 3146 & 35.5 & 94.2 & 1.3 & 0.118 \\
\hline Supp. Vitamin C & 1198 & 95.1 & 220.8 & 8.2 & 3146 & 105.0 & 243.7 & 17.1 & 0.312 \\
\hline Supp. Thiamin (mg) & 1198 & 0.9 & 1.5 & 0.0 & 3146 & 0.9 & 1.6 & 0.0 & 0.042 \\
\hline Supp. Riboflavin (mg) & 1198 & 0.8 & 1.2 & 0.0 & 3146 & 0.9 & 1.3 & 0.0 & 0.042 \\
\hline Supp. Niacin (mg) & 1198 & 9.6 & 13.0 & 0.0 & 3146 & 10.5 & 13.8 & 0.3 & 0.052 \\
\hline Supp. Vitamin B6 (mg) & 1198 & 2.3 & 7.4 & 0.0 & 3146 & 2.8 & 8.4 & 0.0 & 0.029 \\
\hline Supp. Folic Acid (mcg) & 1198 & 156.8 & 189.9 & 0.0 & 3146 & 171.7 & 194.1 & 6.6 & 0.020 \\
\hline Supp. Vitamin B12 (mcg) & 1198 & 2.3 & 2.8 & 0.0 & 3146 & 2.5 & 2.8 & 0.1 & 0.020 \\
\hline Supp. Vitamin D (mcg) & 1198 & 66.8 & 199.6 & 0.0 & 3146 & 88.3 & 228.9 & 0.0 & 0.010 \\
\hline Supp. Calcium (mg) & 1198 & 34.2 & 45.6 & 0.0 & 3146 & 37.4 & 46.7 & 0.0 & 0.029 \\
\hline Supp. Magnesium (mg) & 1198 & 6.7 & 9.0 & 0.0 & 3146 & 7.4 & 9.4 & 0.0 & 0.022 \\
\hline Supp. Iron (mg) & 1198 & 5.9 & 8.1 & 0.0 & 3146 & 6.5 & 8.5 & 0.0 & 0.029 \\
\hline Supp. Zinc (mg) & 1198 & 0.7 & 0.9 & 0.0 & 3146 & 0.7 & 0.9 & 0.0 & 0.029 \\
\hline Supp. Copper (mg) & 1198 & 151.4 & 185.6 & 0.0 & 3146 & 166.1 & 189.4 & 6.6 & 0.020 \\
\hline Supp. Selenium (mcg) & 1198 & 1.1 & 6.8 & 0.0 & 3146 & 1.4 & 7.6 & 0.0 & 0.242 \\
\hline \multicolumn{10}{|l|}{ Supplement Users Only } \\
\hline Supp. Vitamin A (IU) & 568 & 4362.9 & 2482.6 & 5000.0 & 1589 & 4423.2 & 2373.4 & 5000.0 & 0.415 \\
\hline Supp. Vitamin A (mcg RAE) & 568 & 1308.9 & 744.8 & 1500.0 & 1589 & 1327.0 & 712.0 & 1500.0 & 0.415 \\
\hline Supp. Beta Carotene & 564 & 509.4 & 236.2 & 600.0 & 1587 & 520.2 & 268.8 & 600.0 & 0.653 \\
\hline Supp. Vitamin E & 603 & 67.5 & 120.6 & 20.1 & 1665 & 67.0 & 121.0 & 20.1 & 0.690 \\
\hline Supp. Vitamin C & 652 & 174.8 & 275.2 & 60.0 & 1768 & 186.8 & 300.7 & 60.0 & 0.890 \\
\hline Supp. Thiamin (mg) & 573 & 1.8 & 1.8 & 1.5 & 1603 & 1.8 & 1.9 & 1.5 & 0.363 \\
\hline Supp. Riboflavin (mg) & 573 & 1.7 & 1.3 & 1.7 & 1603 & 1.8 & 1.3 & 1.7 & 0.363 \\
\hline Supp. Niacin (mg) & 583 & 19.8 & 12.2 & 20.0 & 1628 & 20.3 & 12.9 & 20.0 & 0.492 \\
\hline Supp. Vitamin B6 (mg) & 579 & 4.8 & 10.1 & 2.0 & 1621 & 5.4 & 11.0 & 2.0 & 0.217 \\
\hline Supp. Vitamin B12 (mcg) & 571 & 329.0 & 137.8 & 400.0 & 1610 & 335.6 & 136.4 & 400.0 & 0.244 \\
\hline Supp. Folic Acid (mcg) & 558 & 4.9 & 2.0 & 6.0 & 1574 & 5.0 & 1.9 & 6.0 & 0.185 \\
\hline Supp. Vitamin D (mcg) & 241 & 331.9 & 332.1 & 250.0 & 738 & 376.4 & 339.2 & 285.7 & 0.069 \\
\hline Supp. Calcium (mg) & 489 & 83.7 & 30.8 & 100.0 & 1394 & 84.4 & 30.9 & 100.0 & 0.466 \\
\hline Supp. Magnesium (mg) & 503 & 15.8 & 6.7 & 18.0 & 1431 & 16.3 & 7.1 & 18.0 & 0.260 \\
\hline Supp. Iron (mg) & 505 & 13.9 & 6.6 & 15.0 & 1437 & 14.1 & 7.0 & 15.0 & 0.516 \\
\hline Supp. Zinc (mg) & 489 & 1.7 & 0.6 & 2.0 & 1394 & 1.7 & 0.6 & 2.0 & 0.466 \\
\hline Supp. Copper (mg) & 558 & 325.0 & 132.2 & 400.0 & 1574 & 331.9 & 129.1 & 400.0 & 0.185 \\
\hline Supp. Selenium (mcg) & 31 & 42.9 & 0.0 & 42.9 & 103 & 42.9 & 0.0 & 42.9 & 1.000 \\
\hline
\end{tabular}

*N refers to the number of participants.

\section{Acknowledgements}

This research was funded in part by grant 1UL1RR025011 from the Clinical and Translational Science Award (CTSA) program of the National Center for Research Resources, National Institutes of Health. The authors acknowledge the contributions of Cathy Schneider and Carla Rottscheit to data collection and management.

\section{Author details}

${ }^{1}$ Center for Human Genetics, Marshfield Clinic Research Foundation, $1000 \mathrm{~N}$. Oak Ave (MLR), Marshfield, WI 54449, USA. ${ }^{2}$ Biomedical Informatics Research Center, Marshfield Clinic Research Foundation, 1000 N. Oak Ave (ML8), Marshfield, WI 54449, USA. ${ }^{3}$ Epidemiology Research Center, Marshfield Clinic Research Foundation, 1000 N. Oak Ave (ML2), Marshfield, WI 54449, USA.

\section{Authors' contributions}

LS assisted in the interpretation of the data and drafted the manuscript. RB conducted the statistical analyses and assisted with data interpretation. DC assisted with data interpretation. WF collected the data. TK collected the data. LC assisted with study design and data interpretation. CAM was the Principal Investigator, contributing to all aspects of the project. All authors were involved in revising the manuscript and reviewed and approved the final version of the manuscript.

\section{Competing interests}

The authors declare that they have no competing interests.

Received: 12 August 2010 Accepted: 28 January 2011 Published: 28 January 2011 


\section{References}

1. Tucker KL: Assessment of usual dietary intake in population studies of gene-diet interaction. Nutr Metab Cardiovasc Dis 2007, 17:74-81.

2. Lussier-Cacan S, Bolduc A, Xhignesse M, Niyonsenga T, Sing CF: Impact of alcohol intake on measures of lipid metabolism depends on context defined by gender, body mass index, cigarette smoking, and Apolipoprotein E genotype. Arterioscler Thromb Vasc Biol 2002, 22:824-831.

3. Subar AF, Harlan LC, Mattson ME: Food and nutrient intake differences between smokers and non-smokers in the US. Am J Publ Health 1990, 80:1323-1329.

4. Talmund PJ, Humphries SE: Gene:Environment interactions and coronary heart disease risk. World Rev Nutr Diet 2004, 93:29-40.

5. Talmud PJ: How to identify gene-environment interactions in a multifactorial disease: CHD as an example. Proc Nutr Soc 2004, 63(1):5-10.

6. Eichner JE, Kuller LH, Ferrell RE, Meilahn EN, Kamboh MI: Phenotypic effects of apolipoprotein structural variation on lipid profiles. III. Contribution of apolipoprotein E phenotype to prediction of total cholesterol, apolipoprotein B, and low density lipoprotein cholesterol in the healthy women study. Arteriosclerosis 1990, 10:379-385.

7. Boerwinkle $E$, Utermann $G$ : Simultaneous effects of the apolipoprotein $E$ polymorphism on apolipoprotein $E$, apolipoprotein $B$, and cholesterol metabolism. Am J Hum Genet 1988, 42:104-112.

8. McCarty CA, Wilke RA, Giampietro PF, Wesbrook SD, Caldwell MD: The Marshfield Clinic Personalized Medicine Research Project (PMRP): design, methods and recruitment for a large population-based biobank. Personalized Med 2005, 2:49-79.

9. McCarty CA, Mukesh BN, Giampietro PF, Wilke RA: Healthy People 2010 prevalence in the Marshfield Clinic Personalized Medicine Research Project: opportunities for public health genomics research. Personalized Med 2007, 4:183-190.

10. McCarty CA, Peissig P, Caldwell MD, Wilke RA: The Marshfield Clinic Personalized Medicine Research Project: 2008 scientific update and lessons learned in the first 6 years. Personalized Med 2008, 5:529-541.

11. Cross DS, Ivacic LC, McCarty CA: Development of a fingerprinting panel using medically relevant polymorphisms. BMC Med Genom 2009, 2:17.

12. Subar AF, Thompson FE, Kipnis V, Midthune D, Hurwitz $P$, McNutt $S$, McIntosh A, Rosenfeld S: Comparative validation of the Block, Willett, and National Cancer Institute food frequency questionnaires. The Eating at America's Table Study. Am J Epidemiol 2001, 154:1089-1099.

13. Schatzkin A, Kipnis V, Carroll RJ, Midthune D, Subar AF, Bingham S, Schoeller DA, Troiano RP, Freedman LS: A comparison of a food frequency questionnaire with a 24-hour recall for use in an epidemiological cohort study: results from the biomarker-based Observing Protein and Energy Nutrition (OPEN) study. In J Epidemiol 2003, 32:1054-1062.

14. Millen AE, Midthune $D$, Thompson FE, Kipnis V, Subar AF: The National Cancer Institute Diet History Questionnaire: validation of pyramid food servings. Am J Epidemiol 2005, 163:279-288.

15. Dixon LB, Subar AF, Wideroff L, Thompson FE, Kahle LL, Potischman N: Carotenoid and tocopheral estimates from the NCl Dietary History Questionnaire are valid compared with multiple recalls and serum biomarkers. J Nutr 2006, 136:3054-3061.

16. Flood A, Subar AF, Hull SG, Zimmerman TP, Jenkins DJA, Schatzkin A: Methodology for adding glycemic load values to the National Cancer Institute Diet History Questionnaire database. J Am Diet Assoc 2006, 106:393-402.

17. Thompson FE, Kipnis V, Midthune D, Freedman LS, Carroll RJ, Subar AF, Brown CC, Butcher MS, Mouw T, Leitzmann M, Schatzkin A: Performance of a food-frequency questionnaire in the US NIH-AARP (National Institutes of Health-American Association of Retired Persons) Diet and Health Study. Publ Health Nutr 2007, 11:183-195.

18. Wright JD, Wang CY, Kennedy-Stephenson J, Ervin RB: Dietary intake of ten key nutrients for public health, United States: 1999-2000. Adv Data 2003, 334:1-4.

doi:10.1186/1475-2891-10-13

Cite this article as: Strobush et al:: Dietary intake in the Personalized Medicine Research Project: a resource for studies of gene-diet interaction. Nutrition Journal 2011 10:13.

\section{Submit your next manuscript to BioMed Central and take full advantage of:}

- Convenient online submission

- Thorough peer review

- No space constraints or color figure charges

- Immediate publication on acceptance

- Inclusion in PubMed, CAS, Scopus and Google Scholar

- Research which is freely available for redistribution 